\title{
Transgenerational epigenetic effects of the endocrine disruptor vinclozolin on pregnancies and female adult onset disease
}

\author{
Eric E Nilsson, Matthew D Anway, Jacob Stanfield and Michael K Skinner \\ Center for Reproductive Biology, School of Molecular Biosciences, Washington State University, Pullman, \\ Washington 99164-4231, USA \\ Correspondence should be addressed to M K Skinner; Email: skinner@wsu.edu
}

\begin{abstract}
Endocrine disruptor exposure during gonadal sex determination was previously found to induce male rat adult onset transgenerational disease (F1-F4 generation), and this was associated with an alteration in the epigenetic (i.e., DNA methylation) programming of the male germ line. The current study was designed to characterize the transgenerational disease phenotypes of the female adult offspring. Pregnant rats (F0 generation) were treated transiently with vinclozolin (i.e., fungicide with anti-androgenic activity) on embryonic (E) days E8-E14 of gestation. F1 control and vinclozolin generation offspring from different litters were mated to produce F2 offspring, and similarly F2 generation animals produced F3 generation offspring. Observations demonstrated that 9 out of 105 pregnant rats (8.6\%) from the vinclozolin F1-F3 generations exhibited uterine hemorrhage and/or anemia late in pregnancy. None (0 out of 82) of the control F1-F3 generation females had similar pregnancy problems. Complete blood cell counts and serum chemistry profiles demonstrated that selected vinclozolin generation animals, but not controls, exhibited marked regenerative anemia in late pregnancy. Examination of kidney histology revealed moderate or severe glomerular abnormalities in $67 \%$ of the vinclozolin F2 and F3 generation adult females compared with $18 \%$ of the controls. Adult female vinclozolin generation animals also developed various types of tumors in $6.5 \%$ of the animals (11 out of 170), while $2 \%$ of control-line animals ( 3 out of 151) developed mammary tumors. Observations demonstrate that vinclozolin exposure during gonadal sex determination promotes a transgenerational increase in pregnancy abnormalities and female adult onset disease states.

Reproduction (2008) 135 713-721
\end{abstract}

\section{Introduction}

Exposure of a pregnant female to an environmental toxicant can lead to the development of abnormalities or disease states in the F1 generation offspring. Although embryonic or early postnatal exposure to radiation or chemical mutagens can promote mutations in the DNA sequence at low frequency (i.e., $0.01 \%$; Barber et al. 2002), most environmental compounds are not mutagens. The fetal basis of adult onset disease has been well documented (Gluckman \& Hanson 2004, Reynolds et al. 2007), but the molecular mechanisms involved are poorly understood and do not appear to involve DNA sequence mutations. In addition to environmental compound exposure, caloric restriction during pregnancy (Zambrano et al. 2005) and fetal exposure to diethylstilbestrol or dexamethasone (Blatt et al. 2003, Drake et al. 2005) can cause abnormalities in the F1 and F2 generations. Both the F1 generation embryo and F2 generation germ line are directly exposed when an F0 generation pregnant mother is exposed. Therefore, the F3 generation is the first unequivocal transgenerational generation. Recently, pregnant female rats exposed during the time of fetal sex determination to the endocrine disruptor vinclozolin have been shown to exhibit in their male offspring transgenerational disease states leading to spermatogenic defects, prostate disease, kidney disease, immune system abnormalities, hypercholesterolemia, and an increased rate of tumor development in the F1-F4 generation offspring (Anway et al. 2005, 2006a, 2006b, Anway \& Skinner 2006, Chang et al. 2006).

The transgenerational disease states in the vinclozolin F1-F4 generation animals were found to be associated with a transgenerational alteration in the epigenetic (i.e., DNA methylation) programming of the male germ line (Anway et al. 2005, Chang et al. 2006). It is hypothesized that reprogramming the male germ line epigenome in subsequent generations is a mechanism by which exposure to the endocrine disruptor vinclozolin can cause a transgenerational phenotype. Epigenetic mechanisms involving DNA methylation have been shown to influence several disease states (reviews: Bjornsson et al. 2004, Egger et al. 2004). An example is that abnormal methylation of genes regulating cytokines can influence the inflammatory component of developing atherosclerosis (Corwin 2004). In addition, 
a change in the methylation state of the insulin-like growth factor-2 gene has been shown to increase the rate of tumor formation (Sakatani et al. 2005).

The current study was designed to investigate the actions of the endocrine disruptor vinclozolin on pregnant F0 rats and the development of transgenerational adult onset disease in their female progeny. Vinclozolin is a fungicide used in agricultural fruit crops, such as grapes for the wine industry (Kelce et al. 1994, Fisher 2004). Vinclozolin is an anti-androgenic compound that is metabolized into further anti-androgenic compounds (Kelce et al. 1994). Embryonic exposure to vinclozolin has been documented to influence sexual differentiation, gonadal formation, and reproductive functions in the exposed F1 generation as they develop and mature (Kelce et al. 1994, Gray et al. 1999, Wolf et al. 2000, Hotchkiss et al. 2002, Uzumcu et al. 2004). As discussed above, vinclozolin exposure during gonadal sex determination promotes a transgenerational epigenetic adult onset disease phenotype in males, which is transmitted through the male germ line for four generations (Anway et al. 2005, 2006a).

Observations were made that some F2 and F3 generation female offspring from the vinclozolin-treated F0 founders developed adult onset disease and had problems with their pregnancies. In late pregnancy, some rats would become pale, lethargic, and occasionally die. The current study was designed to characterize this abnormality in late-stage pregnancy and to document transgenerational adult disease states of females, which occur in the F1-F3 generation offspring of F0 pregnant rats treated with vinclozolin. The ability of an environmental toxicant to promote a variety of different disease states at high frequency for multiple generations suggests a novel epigenetic transgenerational mechanism for disease etiology.

\section{Results}

Pregnant rats were treated with vinclozolin on days E8E14 of gestation, which includes the time of embryonic gonadal sex determination. $\mathrm{F} 1$ generation offspring from different litters were mated to produce F2 offspring, and similarly F2 generation animals produced F3 generation offspring. Only the founder F0 pregnant rats were treated with vinclozolin or received a vehicle control treatment. Observations demonstrated that some F1-F3 vinclozolin generation animals had abnormalities late in their own pregnancies, while no abnormalities were observed in control generation rats. A proportion of vinclozolin generation rats exhibited extreme pallor, lethargy, and sometimes vaginal bleeding in the last 3 days of pregnancy or in the immediate post partum period. These occurrences of pallor and illness did not correlate with the age of the mother at pregnancy (data not shown). In three out of the nine affected females, blood samples were analyzed. In each case, their packed cell volume (PCV) was quite low (PCV $=13,18$, and $22 \%$ respectively), indicating that the pallor and vaginal bleeding seen were associated with anemia. Table 1 shows the incidence of anemia, vaginal bleeding, and pallor in vinclozolin versus control generation rats. Five out of the nine total affected rats were noted as having vaginal or intrauterine hemorrhage, with the rest exhibiting only pallor with either lethargy or death. None of the affected females were from the same litter. In the F1-F3 generations combined, $8.6 \%$ of vinclozolin-line animals showed abnormalities late in pregnancy, while $0 \%$ of control-line animals showed these signs in the study population. This is a significant difference by Fisher's exact test $(P=0.005)$. It should be noted that Fisher's exact test did not detect a significant difference in F1, F2, or F3 generations analyzed separately. Results indicate that the vinclozolin exposure of the F0 generation gestating female promoted a transgenerational (F1-F3) disease phenotype of pregnancy abnormalities in $\sim 9 \%$ of animals ( $n=105$ examined).

F2 generation vinclozolin-line males were bred to wild-type females to produce $\mathrm{F} 3$ generation vinclozolin outcross (VOC) females. Out of 30 VOC females monitored during pregnancy, none developed the anemic illness phenotype described above. Similarly, when F2 vinclozolin-line females were bred to wild-type males to produce F3 reverse VOC (RVOC) offspring, 0 out of 15 RVOC females developed the anemic illness phenotype. This suggests that both male and female parents must be vinclozolin-line animals in order for female offspring to express the pregnancy disease phenotype.

Table 1 Incidence of uterine bleeding and pallor late in pregnancy.

\begin{tabular}{|c|c|c|c|c|c|c|}
\hline \multirow[b]{2}{*}{ Generation } & \multicolumn{3}{|c|}{ Control } & \multicolumn{3}{|c|}{ Vinclozolin } \\
\hline & Number pregnancies & $\begin{array}{c}\text { Number pregnancy } \\
\text { problems }\end{array}$ & $\begin{array}{l}\text { Percent (\%) preg- } \\
\text { nancy problems }\end{array}$ & Number pregnancies & $\begin{array}{l}\text { Number pregnancy } \\
\text { problems }\end{array}$ & $\begin{array}{c}\text { Percent }(\%) \\
\text { pregnancy } \\
\text { problems }\end{array}$ \\
\hline F1 & 42 & 0 & 0 & 53 & 3 & 5.6 \\
\hline F2 & 29 & 0 & 0 & 41 & 5 & 12.2 \\
\hline F3 & 11 & 0 & 0 & 11 & 1 & 9.0 \\
\hline Total (F1-F3) & 82 & 0 & 0 & 105 & 9* & 8.6 \\
\hline
\end{tabular}

A statistical difference was found for the incidence of vinclozolin-line pregnancy problems compared with control $(* P=0.005)$ by Fisher's exact test. 
To better characterize the problems that were occurring with these pregnant rats, blood samples were obtained between day 19 and the day of parturition from a subset of F2 and F3 generation animals and were subjected to a complete blood count, serum chemistry analysis, or manual PCV measurements as described in the 'In vivo procedures and treatment groups' section of the Materials and Methods. Examination of red blood cell PCV revealed that 5 out of 35 vinclozolin generation animals were anemic (Fig. 1). PCV measures the proportion of the blood that is composed of red blood cells. These five animals were derived from three out of the five vinclozolin-treated F0 founder lines examined. None of the affected females were from the same litter. One anemic female was from the F3 generation. Nucleated red blood cells, anisocytosis (variation in red blood cell size), and polychromasia (variation in red blood cell staining) are indicative of a regenerative response to anemia. Out of the 13 vinclozolin generation animals that were subjected to a complete blood count, 2 had nucleated red blood cells present in their complete blood count, while 3 out of the 13 showed anisocytosis and polychromasia in their red blood cell morphology (derived from two out of the three founder lines examined). None of 11 control generation rats showed these abnormalities (representing three control F0 founder lines).

The complete blood count also revealed differences in the proportion of white blood cell types in vinclozolinline compared with control-line rats in late pregnancy. The mean percentage of segmented neutrophils was significantly $(P=0.01)$ higher in vinclozolin-line rats, while the mean percentage of lymphocytes was significantly $(P=0.02)$ lower (Fig. 2$)$. The complete blood count also measured several red blood cell, white blood cell, and platelet parameters for which no significant differences were found between vinclozolin and control F2 and F3 generation rats. Supplementary Table 1, which can be viewed online at www.reproduction-online.org/ supplemental/, presents a list of tests performed for which no differences were found between treatment groups. Therefore, vinclozolin promoted specific blood

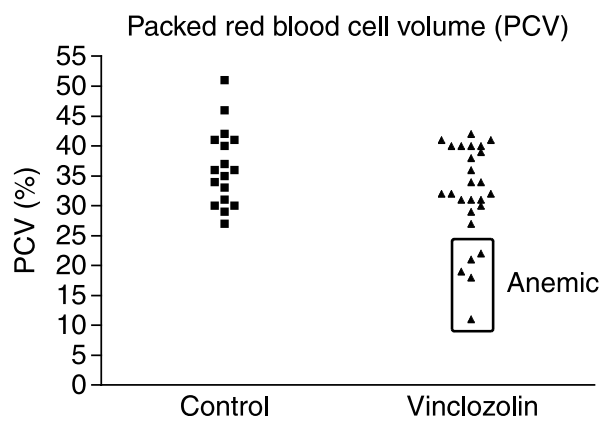

Figure 1 Scatter plot of packed cell volumes (PCV) for individual F2 and F3 generation female rats of control and vinclozolin-line treatment groups from whole blood samples taken at days 19-21 of pregnancy. Boxed points indicate animals with anemia.

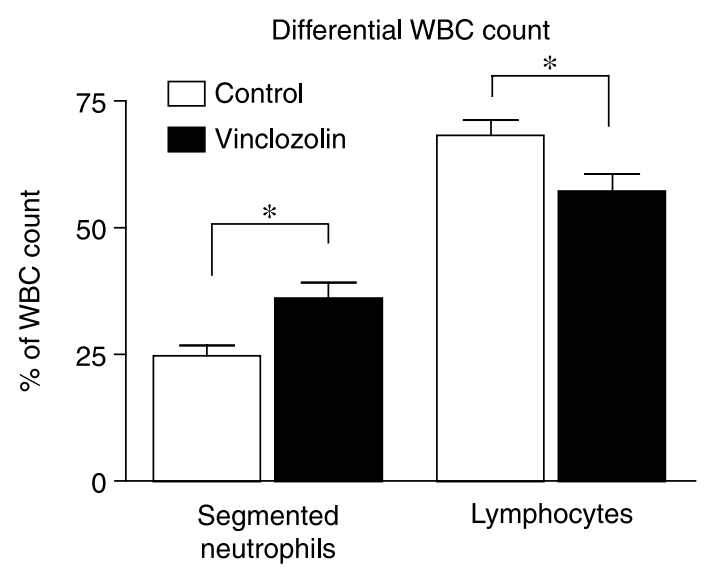

Figure 2 Results of differential white blood cell (WBC) counts for F2 and F3 generation female rats of control and vinclozolin-line treatment groups from whole blood samples taken at days 19-21 of pregnancy. Mean \% values \pm S.E.M. are presented for segmented neutrophils and lymphocytes. $n=8$ (6 F2 + 2 F3), control-line; $n=12$ (10 F2=2 F3), vinclozolin-line animals. An asterisk $(*)$ denotes a significant $(P<0.05)$ difference by Student's $t$-test.

abnormalities, but the majority of the parameters tested were not affected.

Serum chemistry analyses revealed differences between treatment groups for blood urea nitrogen $(B \cup N)$, sodium, and potassium (Table 2). Mean BUN levels are significantly $(P=0.009)$ increased in the serum of F2 and F3 vinclozolin generation rats on days 19-21 of gestation compared with controls. Conversely, mean sodium and potassium plasma levels were significantly $(P=0.009$ and 0.039 respectively) lower in vinclozolin generation rats. Further serum chemistry analyses measured levels of several blood serum components including enzymes, clotting parameters, and hormone levels. No significant differences were found between treatment groups for progesterone or estrogen levels at mid-pregnancy (embryonic day 16). No other significant differences $(P>0.05)$ were found between vinclozolin and control F2 and F3 generation rats for the serum chemistry parameters measured (Supplementary Table 2, which can be viewed online at www.reproductiononline.org/supplemental/). Therefore, the late-stage pregnant vinclozolin generation females did have alterations in selected serum parameters, but the majority were not influenced.

Since BUN, sodium, and potassium levels are markers for abnormal kidney function, and all were found to be different between control and vinclozolin generation rats, kidneys from F2 and F3 generation females were examined histologically for signs of pathology. Kidneys were from 2- to 10-month-old rats, both pregnant and non-pregnant, with the proportion of kidneys from 8- to 10-month-old rats being the same in vinclozolin-line and control-line animals. Observations demonstrate pathological changes in the glomeruli of vinclozolin generation female kidneys. Abnormalities included 
Table 2 Analysis of blood serum from late-stage (days 19-21) gestating female F2 and F3 control and vinclozolin-line rats.

\begin{tabular}{lcrc}
\hline & \multicolumn{2}{c}{ Serum chemistry parameters } \\
\cline { 2 - 4 } & Control line (mean \pm S.E.M.) & Vinclozolin line (mean \pm S.E.M.) & $P$ value \\
\hline Blood urea nitrogen $(\mathrm{mg} / \mathrm{dl})$ & $19.6 \pm 0.7(n=9)$ & $27.2 \pm 2.6(n=14)$ & 0.027 \\
Sodium $(\mathrm{mEq} / \mathrm{l})$ & $150.4 \pm 1.5(n=8)$ & $143.8 \pm 1.4(n=13)$ & 0.006 \\
Potassium $(\mathrm{mEq} / \mathrm{l})$ & $6.9 \pm 0.4(n=8)$ & $6.0 \pm 0.2(n=13)$ & 0.016 \\
\hline
\end{tabular}

Two samples from F3 generation females are in each treatment group, with the remaining being F2. Differences between treatment group means were tested using $t$-test with Welch's correction for non-equal variances, with $P<0.05$ considered significant.

partial or circumferential thickening of Bowman's capsules surrounding glomeruli (Fig. 3). It was found that 2 out of the $11(18 \%)$ of control F2 and F3 generation females were moderately or severely affected, as defined in the Materials and Methods section, while 10 out of the $15(67 \%)$ of vinclozolin generation females were similarly affected (significant difference by Fisher's exact test, $P<0.05$ ). Therefore, the vinclozolin generation females had an increase in kidney abnormalities as determined with both serum and histological analyses.

Blood loss from uterine hemorrhage due to placental abruption is occasionally a complication of preeclampsia in human pregnancies (Sibai 2003). Hallmarks of human pre-eclampsia include hypertension and proteinuria (abnormal levels of protein in urine; Sibai 2003). Since rodent models of pre-eclampsia are desirable for research, it was decided to investigate whether the pregnancy abnormalities in vinclozolin generation rats were similar to human pre-eclampsia. Blood pressure was measured in both younger (2-6


Figure 3 Representative histological sections of female rat pathologies. (A) Kidney from control-line rat showing normal glomerular architecture. Arrow indicates Bowman's capsule with normal thickness. (B) Kidney from vinclozolin-line rat showing glomerular abnormalities. Arrow indicates mild fibrosis and thickened Bowman's capsule. (C) Mammary tumor (mammary fibroadenoma) from a vinclozolin-line rat. (D) Pituitary tumor (pituitary adenoma) from a vinclozolin-line rat. Arrow indicates a band of normal pituitary tissue at the edge of the expansive tumor. months) and older (7-10 months) control, VOC and vinclozolin generation female rats during mid- and late pregnancy, and in non-pregnant animals. No significant differences were detected in the mean blood pressure of F2 and F3 vinclozolin generation females rats when compared with control females at any age, either pregnant or non-pregnant (Supplementary Table 3, which can be viewed online at www.reproductiononline.org/supplemental/).

The incidence of tumor formation was recorded in vinclozolin and control F1-F3 generation adult female rats. In the experimental population, 11 out of the 170 vinclozolin generation females developed tumors, compared with 3 out of 151 control generation females. This is not a statistically significant difference $(P=0.10)$ by Fisher's exact test. The majority of the tumors identified were mammary tumors (Fig. 3C), encompassing mammary fibroadenomas, mammary adenomas, and mammary adenocarcinomas. Non-mammary tumors occurred only in the vinclozolin-treated lines, including one cerebral astrocytoma and two pituitary adenomas (Fig. 3D).

\section{Discussion}

The F1-F3 generation female progeny of gestating female rats exposed to vinclozolin during gonadal sex determination were found to have serious abnormalities (anemia) late in their pregnancies, while control F1-F3 generation females had no anemic abnormalities. The non-parametric Fisher's exact test was not sensitive enough to detect a statistical difference in the incidence of pallor and illness between treated and control-line animals when each generation (F1-F3) was analyzed separately. However, when the data were pooled, a significant $(P=0.005)$ difference was revealed. In such cases, there is a concern that an effect of generation may confound the analysis. No intergenerational differences or effects were observed with any of the other analyses in this study, but the low numbers make this a variable to consider in data interpretation. Also, there were no cases of pallor or illness in control females. This suggests that pregnancy problems of anemic illness are a result of ancestral vinclozolin exposure. Affected anemic animals were not from the same litter, but were from several F0 founder lines. In fact, all three affected F1 generation 
animals were from different F0 treated mothers, suggesting that this is not an effect of a particular genetic line. The anemia phenotype occurred in an F3 generation female that was not herself directly exposed to vinclozolin. Therefore, this abnormal late-stage pregnancy phenotype is transgenerational. Such transgenerational phenotypes have previously been shown to involve epigenetic modifications of the germ line (Anway et al. 2005). Further characterization of this adult onset disease phenotype in a subset of F2 and F3 generation rats revealed that anemic females were mounting a regenerative response to replace their lost red blood cells. This was demonstrated by the presence of nucleated red blood cells, polychromasia, and anisocytosis in blood samples from anemic animals. These findings show that bone marrow production of red blood cells is normal and indicates that the erythrocyte loss is chronic or recurrent over at least the later part of the pregnancy. If there had been only a single event of blood cell loss in the last few days of pregnancy, there would have been no time for bone marrow to increase the rate of erythrocyte production and have the complete blood count show the characteristic signs of a regenerative response. Interestingly, these particular females exhibited no external signs of hemorrhage earlier in their pregnancies. Also, many anemic rats showed no sign of uterine hemorrhage upon necropsy, suggesting that red blood cell loss may be hemolytic. However, for some females showing pallor and lethargy late in pregnancy, necropsy did show uterine hemorrhage. Further studies will be needed to definitively determine the cause of chronic blood loss in these transgenerational pregnant females.

Blood analyses of F2 and F3 generation pregnant females revealed that the mean percentage of lymphocytes was decreased and that of neutrophils increased in vinclozolin generation females compared with controls. This is consistent with a 'stress leukogram'. A stress leukogram is a decrease in the number of circulating blood lymphocytes and a corresponding increase in the percentage of segmented neutrophils, which occur when an animal experiences chronic or repeated physiological stress (Stockham \& Scott 2002). Since the same set of blood cell samples for 15 different parameters were used for the complete blood analysis, a multiple testing correction suggests that the significance levels ( $P$ values) listed above must be viewed with caution. However, the fact that low lymphocyte and high neutrophil levels are consistent with the physiological condition known as stress leukogram, the finding appears physiologically relevant. The anemia phenotype seen to develop in pregnant vinclozolin generation females could easily be enough of a stressor to induce the changes in white blood cell proportions observed. Alternatively, the underlying condition that leads to the anemia phenotype might induce a stress leukogram. Therefore, the blood analysis and stress leukogram correlate with the pregnancy abnormality observed.

The blood serum chemistry profiles of F2 and F3 generation pregnant females revealed that the mean BUN, sodium, and potassium levels were significantly different $(P=0.009,0.009$, and 0.039 respectively) in vinclozolin generation females compared with controls. Similar to the complete blood cell count analysis, the same set of serum samples were evaluated for 14 different serum chemistry parameters. A multiple testing correction suggests that the $P$ values above, especially those for serum potassium $(P=0.039)$, be viewed with caution. However, all these blood parameters are influenced by kidney function, which appear physiologically relevant. An increase in BUN, as was seen in vinclozolin generation females, suggests there are changes in the glomerular filtration that occurs in the kidneys. Therefore, the kidneys of F2 and F3 generation rats were examined histologically for signs of pathological changes. In addition to the analysis of pregnant females, the kidneys of non-pregnant females were also examined. In $\sim 70 \%$ of the vinclozolin generation females, the kidneys had a thickening of Bowman's capsules surrounding glomeruli (Ronco \& Debiec 2006) in comparison with $<20 \%$ in control generation females. These glomerular changes as scored for this study (see the Materials and Methods section) were mild and likely would not have resulted in complete kidney failure. Although such changes are often seen in aging rats, the age distribution of the females from whom kidneys were examined was similar in the vinclozolin and control population animals (2-10 months; data not shown). The higher proportion of abnormal kidney pathologies seen in vinclozolin generation females suggests a more rapid progression of this adult onset disease. Therefore, the kidney pathology observed correlated with the changes in BUN, sodium, and potassium seen in vinclozolin generation females. This adult onset kidney disease was seen in both nonpregnant and pregnant females.

The majority of the blood and serum chemistry parameters tested were not significantly different between control and vinclozolin generation animals (Supplementary Tables 1 and 2). For many analyses, the data for F2 and F3 generation animals were combined. A limitation of the current study is that fewer F3 generation animals were used than F2 generation animals. Major generational effects were not observed as described previously (Anway et al. 2006b), and similar observations were made between the F2 and F3 generations. Therefore, the combined F2 and F3 generation data were used to assess transgenerational phenotypes. Statistical analysis of the F1 generation animals can consider either the individual animal or litter as the experimental unit, but transgenerational F2 and F3 animals require individual animals to be the experimental unit. This accounts for biological variation 
between animals and phenotypes that are independent of the litter exposure.

Previous studies have demonstrated that after exposure of a pregnant F0 rat to vinclozolin during gonadal sex determination, an adult onset spermatogenic cell defect (i.e., apoptosis) developed transgenerationally in F1-F4 generation males (Anway et al. 2005, 2006a, 2006b, Anway \& Skinner 2006, Chang et al. 2006). This transgenerational adult onset disease occurred in the next generation even when vinclozolin generation males were mated with control generation females. By contrast, this transgenerational spermatogenic defect phenotype was not transmitted to the next generation if vinclozolin generation females were mated with control males (Anway et al. 2005, 2006b). In the current study, it was found in 30 VOC and 15 RVOC matings that both the female and male rats had to be from vinclozolin-exposed lines in order for the anemia phenotype to occur in pregnant females of the subsequent generation. While a larger study population would be more definitive, this suggests that the proposed transgenerational epigenetic phenotype of adult onset disease induction may be different for the development of anemia during pregnancy than for those changes that influence the induction of other disease states. Results imply that the 'inheritance' of disease-inducing epigenetic changes is complex. Previous studies have also suggested a complex epigenetic inheritance of disease. In a study of hereditary nonpolyposis colorectal cancer, it was found that abnormal hypermethylation of one allele of the DNA mismatch repair protein $(M L H 1)$ gene was associated with predisposition to the cancer. Inheritance of the affected maternal chromosome in some cases resulted in offspring also showing abnormal $M L H 1$ methylation, while in other cases $\mathrm{MLH} 1$ methylation had reverted to normal (Hitchins et al. 2007). The factors that determine the inheritance of epigenetic changes are not yet known.

The signs of blood loss late in pregnancy and the glomerular pathology of the kidneys that were seen in pregnant rats in the current study are also clinical parameters of the human pregnancy disease condition pre-eclampsia (Roberts \& Lain 2002, Redman \& Sargent 2005, Sibai 2003, Signore et al. 2006). Another prominent parameter of human pre-eclampsia is hypertension late in pregnancy. Blood pressure was measured in the pregnant rats from F2 and F3 generation vinclozolin and control females in order to see whether the pregnancy phenotype observed in the vinclozolin generation rats closely resembled pre-eclampsia in humans. Blood pressure values were not significantly different in vinclozolin generation females, suggesting that this complex of kidney pathology and anemia seen in transgenerational vinclozolin pregnant rats may not be the same as human pre-eclampsia. Alternatively, physiological defects associated with the development of human pre-eclampsia may occur in the rats, but rats may not express hypertension as a response. Other markers of pre-eclampsia, such as platelet count and liver enzyme levels (alanine aminotransferase and alkaline phosphatase), were also not different between vinclozolin and control generation animals (Supplementary Tables 1 and 2). Further studies are necessary to elucidate the pathophysiological cause of this disease condition that occurs transgenerationally in the offspring of vinclozolin generation females.

Previous studies of the transgenerational epigenetic actions of vinclozolin on adult onset disease in males showed an increase in the rate of tumor formation in aging vinclozolin F1-F4 generations (Anway et al. 2006a). In the current study, the incidence of tumors was found to be $6.5 \%$ in vinclozolin generation animals when compared with $2 \%$ in control generation animals. This incidence of tumor formation in vinclozolin-line females is similar to what was seen in vinclozolin generation males (Anway et al. 2006a). However, in vinclozolin-line females, this $6.5 \%$ tumor incidence was not statistically different than the $2 \%$ tumor incidence seen in control-line females ( $P=0.10$ by Fisher's exact test). Therefore, one cannot conclude that vinclozolin-line females are more prone to tumor formation than controls. In previous studies of vinclozolin-line male rats versus controls, the control-line study population had essentially 0\% tumor incidence (Anway et al. 2006a). Aging female Sprague-Dawley rats are prone to spontaneous mammary tumor formation with mammary tumor incidence being as high as $60 \%$ in very old (105 weeks) females (Gitkins \& Clifford 2004, Charles River Laboratories report, www.criver.com/flex_content_area/documents/rm_rm_r_lesions_survival_crlcd_sd_rats.pdf). All of the tumors seen in the current study in control-line animals were mammary tumors. Therefore, it is possible that any effect of vinclozolin treatment to increase tumor incidence was lost in the 'noise' of spontaneous mammary tumor formation in female rats. Further investigations with a larger study population may be needed to definitively determine whether vinclozolin treatment has an effect on mammary tumor incidence in females. Interestingly, other tumors (e.g. pituitary tumors) did develop in vinclozolin generation females but not in control generation females. These observations suggest the vinclozolin generation females, as observed in the males (Anway \& Skinner 2006), may have an increased incidence of adult onset tumor formation.

The transgenerational disease phenotype in males is associated with transgenerational alterations in the epigenetic (i.e., DNA methylation) patterning of the male germ line (Anway et al. 2005, Anway \& Skinner 2006, Chang et al. 2006). Although epigenetic mechanisms were not evaluated in the current study, similar transgenerational epigenetic changes are anticipated to lead to the disease phenotypes seen in vinclozolin generation female rats. When the pregnant females are exposed to vinclozolin, both the F1 generation embryos and the germ line cells that will form the F2 generation zygotes are also directly exposed 
to vinclozolin. Since F3 generation females show the disease phenotypes of anemia during pregnancy, white blood cell abnormalities, serum chemistry changes, and kidney abnormalities, observations indicate that the vinclozolin exposure can induce transgenerational disease states. Although the testis phenotype is transmitted through the male germ line, both male and female parents had to be of vinclozolin-exposed lines in order for female offspring to show the pregnancy disease phenotype. The extent to which the female germ line is involved in the epigenetic transgenerational effects of vinclozolin exposure remains to be elucidated.

In summary, exposure of pregnant rats to the endocrine disruptor vinclozolin resulted in the development of adult onset disease states in their female progeny. The adult onset transgenerational disease states characterized included severe anemia; a decreased percentage of lymphocytes along with an increased percentage of neutrophils; changes in serum BUN, sodium, and potassium levels; and an increased rate of renal glomerular pathology. This phenotype developed transgenerationally in females of the F1-F3 generations. These results support the findings of a transgenerational epigenetic disease phenotype in animals exposed during embryonic gonadal development to the environmental toxicant vinclozolin (Anway et al. 2005, 2006a, 2006b, Anway \& Skinner 2006, Chang et al. 2006). These findings also are consistent with the hypothesis that the multi-generational effects of exposure to diethylstilbestrol, dexamethasone, or caloric restriction during pregnancy (Blatt et al. 2003, Drake et al. 2005, Zambrano et al. 2005) are in part due to epigenetic alterations. The ability of an environmental toxicant to promote a variety of different disease states for multiple generations supports the idea that a novel mechanism for disease etiology exists involving epigenetic transmission.

\section{Materials and Methods}

\section{In vivo procedures and treatment groups}

Pregnant outbred Sprague-Dawley rats housed at a Washington State University Vivarium were given daily i.p. injections of vinclozolin (100 mg/kg per day) from embryonic days 8 to 14 (E8-E14) of gestation as described previously (Cupp et al. 2003). These pregnant rats were designated as F0 generation animals. The sperm-positive vaginal smear date was considered to be embryonic day 0 . Pregnant control mothers received vehicle alone (i.e., DMSO and sesame oil). Six different founder lines (individual F0 injected gestating females) were generated for controls and eight different borderlines were generated for vinclozolin-treated groups for these analyses. Offspring of these vinclozolin-treated pregnant mothers were designated as F1 animals ( $n=53$ females). F1 vinclozolin generation males were bred to $\mathrm{F} 1$ vinclozolin generation females to generate the F2 vinclozolin generation $(n=41)$, and F2 vinclozolin males were bred to F2 vinclozolin females to generate the F3 generation $(n=11)$. Rats for the control groups were bred in the same manner for all the generations (F1: $n=42, \mathrm{~F} 2: n=29, \mathrm{~F} 3: n=11$ ). Note that only the F0 generation pregnant females received vinclozolin or vehicle control injections. No inbreeding or sibling crosses were generated. F1-F3 generation female rats were between 2 and 10 months old when they were bred. Each time a group of vinclozolin generation females were bred, the age-matched control-line females of the same generation were also bred. Pregnant rats were monitored for signs of illness late in their pregnancies. Specifically, it was recorded whether a pregnant female showed extreme pallor (pale eyes, white skin on ears and feet) or vaginal bleeding with pallor and lethargy. Data on tumor incidence were drawn from a larger population of vinclozolin generation (170) and control generation (157) females because tumor incidence had been monitored for a longer time than the anemia phenotype. Tumors were detected initially if clinical signs were present in a female rat, such as s.c. masses on the abdomen (mammary tumors) or lethargy and circling behavior (brain tumors). Clinical signs were followed up by either mass biopsies or necropsies and the identification of tumors by veterinary pathologists at the Washington Animal Diagnostic Disease Laboratory, Washington State University. A VOC experiment involved breeding an F2 vinclozolin generation male with a wild-type female to generate an F3 generation VOC. Wild type is defined as being of the same Sprague-Dawley strain but not from the control generation population. A RVOC experiment involved breeding an F2 vinclozolin generation female with a wild-type male to generate an F3 generation RVOC. The pregnancies of VOC and RVOC female rats were monitored for occurrences of anemia and illness late in gestation.

Blood samples from 11 control rats and 13 rats from vinclozolin-treated lines (representing 3 controls and 3 vinclozolin Fo founder lines) were subjected to a complete blood count and serum chemistry analysis. PCV was measured manually as described in the Materials and Methods section for an additional 18 control-line and 21 vinclozolin-line F2 and F3 generation females to result in a total of 29 control-line and 34 vinclozolin-line animals examined (representing 5 controls and 5 vinclozolin F0 founder lines). PCV data were collected only from matched groups of control-line and vinclozolin-line females prospectively chosen for PCV analysis. For this reason, only two out of the nine females that showed the pallor and vaginal bleeding phenotype (Table 1) were included in the PCV analysis (Fig. 1), even though three out of these nine females had PCV measurements performed. All of the abovementioned rats were of the 82 control-line and 105 vinclozolin-line rats monitored for abnormalities of pregnancy (representing 6 controls and 8 vinclozolin F0 founder lines). The animals were killed by exposing them to $\mathrm{CO}_{2}$ overdose followed by cervical dislocation in unconscious animals. The Washington State University Animal Care and Use Committee approved all procedures.

\section{Blood cell and serum chemistry analyses}

Blood analyses were performed by the Clinical Pathology Laboratory at the Washington State University Veterinary Teaching Hospital with standard procedures previously 
described. For the blood counts (red and white blood cells), data were collected on a Horiba ABX Hematology Analyzer System $910+C P$, with all differential analysis being done manually. In some cases, packed red blood cell volume (PCV) was measured in the laboratory of the investigators using the following method: blood from lancing the lateral saphenous vein was collected into heparinized microhematocrit tubes. The tubes were centrifuged for $5 \mathrm{~min}$ in a Damon IEC MB microhematocrit centrifuge (Damon, Needham Hts., MA, USA), and the height of the packed red cells was expressed as a percentage of total fluid height. The metabolic panel/profile was done on a Cobras MIRA Plus Analyzer (Roche). The serum steroid concentrations were determined by RIA at the Center for Reproductive Biology Assay Core Laboratory.

\section{Blood pressure measurements}

Blood pressure was measured in awake rats placed in a restraint tube. The rats were habituated to the restraint conditions by placing each into the restraint tubes three or more times in the days prior to blood pressure measurement, until the rats remained calm in the tubes. Blood pressure was measured using an inflatable tail cuff system in combination with a Model 179 Blood Pressure Analyzer (IITC Life Sciences, Woodland Hills, CA, USA; Kubota et al. 2006). The average of two or three sequential blood pressure measurements was used.

\section{Histology and pathology}

Tissues were fixed in 10\% neutral buffered formalin or Bouin's fixative (Sigma), embedded in paraffin, sectioned, and then stained with hematoxylin and eosin according to standard procedures. Multiple sections were obtained from each tissue sample for comparison to allow a representative section to be selected. The Center for Reproductive Biology, Histology Core Laboratory, and the Washington Animal Disease Diagnostic Laboratory assisted with these procedures. Renal lesions were diagnosed by an increase in morphologically identified glomerular damage exhibiting a visible fibrotic thickening of Bowman's capsule around at least half of the glomerulus, with or without accompanying reduced glomerular area. Kidneys were taken from both pregnant and non-pregnant rats 2-10 months old. Kidney sections were scored as to the extent of glomerular pathology as follows: $(-)=$ slightly or unaffected to $<10 \%$ glomeruli affected; $(++)=$ moderately affected to $10-60 \%$ glomeruli affected; and $(+++)=$ severely affected to $>60 \%$ of glomeruli affected. Note that slightly and moderately affected individuals would likely show no clinical signs of kidney insufficiency due to excess kidney capacity.

\section{Statistical analysis}

Results from vinclozolin and control generation group animals were compared using Student's t-test or $t$-test with Welch's correction for non-equal variances for parametric data and Fisher's exact test for non-parametric data sets. In the case of analyses of combined F2 and F3 data for neutrophils, lymphocytes, BUN, sodium, and potassium, a two-way ANOVA indicated no significant effect of generation.
Therefore, the F3 data were pooled with that of F2 for several parameters. Analyses were performed using GraphPad Prism 4 version 4.01 for Macintosh (GraphPad Software, San Diego, CA, USA).

\section{Acknowledgements}

We acknowledge the expert technical assistance of Nathan Meyer and thank Ms Rochelle Pedersen for the assistance in preparing the manuscript. We thank Dr Charles Leathers, Veterinary Microbiology and Pathology, Washington State University for his helpful comments and critical review of the manuscript. The current address for Dr Matthew Anway is the Department of Biological Sciences, University of Idaho, Moscow, ID 83844-3051. This research was supported by a grant from the NIH, NIEHS to MKS. The authors declare that there is no conflict of interest that would prejudice the impartiality of this scientific work.

\section{References}

Anway MD \& Skinner MK 2006 Epigenetic transgenerational actions of endocrine disruptors. Endocrinology 147 S43-S49.

Anway MD, Cupp AS, Uzumcu M \& Skinner MK 2005 Epigenetic transgenerational actions of endocrine disruptors and male fertility. Science 308 1466-1469.

Anway MD, Leathers C \& Skinner MK 2006a Endocrine disruptor vinclozolin induced epigenetic transgenerational adult-onset disease. Endocrinology 147 5515-5523.

Anway MD, Memon MA, Uzumcu M \& Skinner MK 2006b Transgenerational effect of the endocrine disruptor vinclozolin on male spermatogenesis. Journal of Andrology 27 868-879.

Barber R, Plumb MA, Boulton E, Roux I \& Dubrova YE 2002 Elevated mutation rates in the germ line of first- and second-generation offspring of irradiated male mice. PNAS 99 6877-6882.

Bjornsson HT, Fallin MD \& Feinberg AP 2004 An integrated epigenetic and genetic approach to common human disease. Trends in Genetics 20 350-358.

Blatt J, Van Le L, Weiner T \& Sailer S 2003 Ovarian carcinoma in an adolescent with transgenerational exposure to diethylstilbestrol. Journal of Pediatric Hematology/Oncology 25 635-636.

Chang HS, Anway MD, Rekow SS \& Skinner MK 2006 Transgenerational epigenetic imprinting of the male germline by endocrine disruptor exposure during gonadal sex determination. Endocrinology 147 5524-5541.

Corwin EJ 2004 The concept of epigenetics and its role in the development of cardiovascular disease: commentary on 'new and emerging theories of cardiovascular disease'. Biological Research for Nursing 6 11-16 (discussion 21-3).

Cupp AS, Uzumcu M, Suzuki H, Dirks K, Phillips B \& Skinner MK 2003 Effect of transient embryonic in vivo exposure to the endocrine disruptor methoxychlor on embryonic and postnatal testis development. Journal of Andrology 24 736-745.

Drake AJ, Walker BR \& Seckl JR 2005 Intergenerational consequences of fetal programming by in utero exposure to glucocorticoids in rats. American Journal of Physiology. Regulatory, Integrative and Comparative Physiology 288 R34-R38.

Egger G, Liang G, Aparicio A \& Jones PA 2004 Epigenetics in human disease and prospects for epigenetic therapy. Nature $\mathbf{4 2 9} 457-463$.

Fisher JS 2004 Environmental anti-androgens and male reproductive health: focus on phthalates and testicular dysgenesis syndrome. Reproduction 127 305-315.

Gluckman PD \& Hanson MA 2004 Developmental origins of disease paradigm: a mechanistic and evolutionary perspective. Pediatric Research 56 311-317. 
Gray LE Jr, Ostby J, Monosson E \& Kelce WR 1999 Environmental antiandrogens: low doses of the fungicide vinclozolin alter sexual differentiation of the male rat. Toxicology and Industrial Health 15 48-64.

Hitchins MP, Wong JJ, Suthers G, Suter CM, Martin DI, Hawkins NJ \& Ward RL 2007 Inheritance of a cancer-associated MLH1 germ-line epimutation. New England Journal of Medicine 356 697-705.

Hotchkiss AK, Ostby JS, Vandenburgh JG \& Gray LE Jr 2002 Androgens and environmental antiandrogens affect reproductive development and play behavior in the Sprague-Dawley rat. Environmental Health Perspectives 110 (Suppl 3) 435-439.

Kelce WR, Monosson E, Gamcsik MP, Laws SC \& Gray LE Jr 1994 Environmental hormone disruptors: evidence that vinclozolin developmental toxicity is mediated by antiandrogenic metabolites. Toxicology and Applied Pharmacology 126 276-285.

Kubota Y, Umegaki K, Kagota S, Tanaka N, Nakamura K, Kunitomo M \& Shinozuka K 2006 Evaluation of blood pressure measured by tail-cuff methods (without heating) in spontaneously hypertensive rats. Biological \& Pharmaceutical Bulletin 29 1756-1758.

Redman CW \& Sargent IL 2005 Latest advances in understanding preeclampsia. Science 308 1592-1594.

Reynolds RM, Godfrey KM, Barker M, Osmond C \& Phillips DI 2007 Stress responsiveness in adult life: influence of mother's diet in late pregnancy. Journal of Clinical Endocrinology and Metabolism 92 2208-2210.

Roberts JM \& Lain KY 2002 Recent insights into the pathogenesis of preeclampsia. Placenta 23 359-372.

Ronco P \& Debiec H 2006 New insights into the pathogenesis of membranous glomerulonephritis. Current Opinion in Nephrology and Hypertension 15 258-263.
Sakatani T, Kaneda A, lacobuzio-Donahue CA, Carter MG, de Boom Witzel S, Okano H, Ko MS, Ohlsson R, Longo DL \& Feinberg AP 2005 Loss of imprinting of Igf2 alters intestinal maturation and tumorigenesis in mice. Science 307 1976-1978.

Sibai BM 2003 Diagnosis and management of gestational hypertension and preeclampsia. Obstetrics and Gynecology 102 181-192.

Signore C, Mills JL, Qian C, Yu K, Lam C, Epstein FH, Karamanchi SA \& Levine RJ 2006 Circulating angiogenic factors and placental abruption. Obstetrics and Gynecology 108 338-344.

Stockham SL \& Scott MA 2002 Fundamentals of Veterinary Clinical Pathology, pp 1-610. Ames, IA: lowa State Press.

Uzumcu M, Suzuki H \& Skinner MK 2004 Effect of the anti-androgenic endocrine disruptor vinclozolin on embryonic testis cord formation and postnatal testis development and function. Reproductive Toxicology $\mathbf{1 8}$ 765-774.

Wolf CJ, LeBlanc GA, Ostby JS \& Gray LE Jr 2000 Characterization of the period of sensitivity of fetal male sexual development to vinclozolin. Toxicological Sciences 55 152-161.

Zambrano E, Martinez-Samayoa PM, Bautista CJ, Deas M, Guillen L, Rodriguez-Gonzalez GL, Guzman C, Larrea F \& Nathanielsz PW 2005 Sex differences in transgenerational alterations of growth and metabolism in progeny (F2) of female offspring (F1) of rats fed a low protein diet during pregnancy and lactation. Journal of Physiology 566 225-236.

Received 5 December 2007

First decision 2 January 2008

Accepted 24 January 2008 